\title{
Reduction in sugar-sweetened beverages is not associated with more water or diet drinks
}

\author{
Jenny Veitch ${ }^{1, *}$, Amika Singh ${ }^{2}$, Maartje M van Stralen ${ }^{2}$, Willem van Mechelen ${ }^{2}$, \\ Johannes Brug ${ }^{3}$ and Mai JM ChinAPaw ${ }^{2}$ \\ ${ }^{1}$ Centre for Physical Activity and Nutrition Research, School of Exercise and Nutrition Sciences, Deakin \\ University, 221 Burwood Highway, Burwood, VIC 3125, Australia: ${ }^{2}$ Department of Public and Occupational \\ Health, EMGO Institute for Health and Care Research, VU University Medial Centre, BT, Amsterdam, The \\ Netherlands: ${ }^{3} \mathrm{EMGO}$ Institute for Health and Care Research, VU University Medial Centre, BT, Amsterdam, \\ The Netherlands
}

Submitted 14 December 2009: Accepted 3 September 2010: First published online 29 0ctober 2010

\begin{abstract}
Objective: The Dutch Obesity Intervention in Teenagers (DOiT) is a school-based randomised controlled trial that was effective in decreasing the consumption of sugar-sweetened beverages among adolescents. The present study examined, using mediation analysis, whether this decrease in consumption of sugar-sweetened beverages could be explained by an increase in the consumption of water or diet drinks.

Design: Participants completed a questionnaire about their beverage consumption at baseline and at 8 months (immediately post-intervention), 12- and 20-month follow-ups. A series of multi-level linear regression analyses were performed to examine water and diet drink consumption as potential mediators of the intervention effect on the consumption of sugar-sweetened beverages.

Setting: Eighteen Dutch secondary schools.

Subjects: A total of 747 adolescents (mean age: $12 \cdot 7$ years).

Results: In addition to the DoiT intervention effect of a reduction in the consumption of sugar-sweetened beverages at 8 months $(-284 \mathrm{ml} / \mathrm{d} ; 95 \% \mathrm{CI}-420,-148)$ and 12 months $(-260 \mathrm{ml} / \mathrm{d} ; 95 \% \mathrm{CI}-360,-160)$, there was also a significant reduction in diet drinks at 8 months $(-52 \mathrm{ml} / \mathrm{d} ; 95 \% \mathrm{CI}-89,-16)$. There was no significant difference in water consumption at any follow-up. The decrease in sugar-sweetened beverage consumption could not be explained by an increase in water or diet drink consumption at any time point.

Conclusions: Interventions aimed at reducing sugar-sweetened beverage consumption may be effective without changing consumption of other beverages. Reducing sugar-sweetened beverages was, however, a main message of the DOiT intervention. It is possible that a concomitant promotion of water may have resulted in a greater increase in water intake and replacement of sugar-sweetened beverages with water.
\end{abstract}

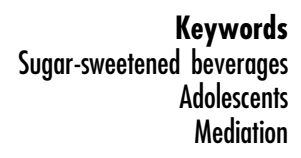

The increased prevalence of obesity among youth is a major public health issue. Childhood obesity is known to track into adolescence and adulthood ${ }^{(1)}$ and is associated with several adverse health outcomes later in life ${ }^{(2)}$.

Low levels of physical activity, and unhealthy diets containing excessive high-energy foods and sugarsweetened beverages, are considered contributors to this worldwide obesity epidemic ${ }^{(3)}$. Soft drink consumption has increased dramatically over recent years ${ }^{(4)}$, and a study of adolescents from twenty-eight European countries has shown that, on average, $26 \%$ of students consume soft drinks on a daily basis ${ }^{(5)}$. There is strong evidence linking soft drink consumption with increased energy intake and body weight ${ }^{(6)}$. US data show that the percentage of total daily energy intake from sweetened beverages (soft drinks and fruit juices) among youth has more than doubled between 1977 and 2001 ${ }^{(7)}$. An unhealthy diet during adolescence may also track into adulthood $^{(8)}$. A focus on decreasing soft drink consumption is therefore likely to be a promising intervention strategy to prevent obesity among youth.

The school-based Dutch Obesity Intervention in Teenagers (DOiT) was effective in decreasing the consumption of sugar-sweetened beverages among adolescents $^{(9,10)}$. Substituting sugar-sweetened beverages with diet drinks and/or water was an objective of the DoiT intervention. The purpose of the present study was to examine whether adolescents who decreased their 
consumption of sugar-sweetened beverages replaced their sugar-sweetened beverages with an increased consumption of water or diet drinks.

\section{Methods}

The present study was nested within DOiT, a schoolbased cluster randomised controlled trial conducted in the Netherlands. Information on the study design and recruitment protocol has been described previously ${ }^{(11)}$. In summary, eighteen secondary schools located within $150 \mathrm{~km}$ of Amsterdam participated in this obesity prevention intervention. These schools selected three classes of first-year students (aged 12-13 years) who received information about the study. Students and their parents gave written informed consent ( $74 \%$ response rate). The ethics committee of the VU University Medical Centre approved the study protocol. Schools were randomly assigned to either the intervention or control group (ten intervention and eight control schools). The DoiT intervention included eleven lessons in the biology and physical education curriculum.

The first part of the intervention was aimed at raising awareness with regard to energy balance-related behaviours (i.e. sugar-sweetened beverage consumption, snack consumption, sedentary behaviour and physical activity). The second part of the intervention aimed at the facilitation of behavioural changes. Assisted by the teachers and intervention materials (worksheets, computerised tailored advice), the adolescents set personal goals, formulated intentions, identified possible barriers, improved their selfefficacy and evaluated change processes. The reduction of sugar-sweetened beverages was strongly promoted as one of the main messages of the DoiT intervention and the advantages of water consumption was repeatedly communicated. Schools were provided with advice on school canteens, addressing both (i) increasing possibilities of a healthier choice, but also on (ii) making the unhealthy choice more difficult. Control schools were required to maintain their regular curriculum.

\section{Measures}

All measures were completed at school, during class time, at the beginning of the school year in 2003 (baseline). They were repeated after 8 months (immediately postintervention), 12 months ( 4 months post-intervention) and 20 months (12 months post-intervention). The adolescents completed a questionnaire in which they were required to self-report their sex, age and parents' country of birth. The participants were also asked to indicate on how many days per week (for a usual week) they consumed three types of beverages: (i) sugar-sweetened beverages (i.e. soft drinks and fruit juice); (ii) diet beverages (i.e. soft drinks with no added sugar such as diet coke); and (iii) water. They also specified the amount per number of servings of each of these beverages they usually consumed on these days. Frequency and quantity were multiplied to obtain estimates of mean daily consumption. These items were adapted from a fruit (including fruit juice), vegetable and fat questionnaire, which were previously shown to be valid and reliable ${ }^{(12,13)}$. To assist the participants to recognise each of these types of beverages, pictures of the beverage were included in the questionnaire. Reported consumption above the 95th percentile was recorded as the value of the 95th percentile. Research assistants were not blinded to the group assignment because they were involved in arranging and conducting the measurements, and delivering the intervention materials; however, by performing all measurements according to a standardised protocol, the potential for observer bias was minimised.

\section{Statistical analyses}

Only adolescents with complete sugar-sweetened beverage data at baseline and at 8 months post-intervention were included in the analyses. Descriptive and exploratory statistics examined characteristics of the sample, differences between the control and intervention groups and the percentage of change in the consumption of beverages from baseline. Multi-level linear regression analyses (using MLwiN version $2 \cdot 14$; Centre for Multilevel Modelling, University of Bristol, Bristol, UK) were performed to examine differences in baseline consumption of sugar-sweetened beverages, water and diet drinks between the intervention and control groups. Three levels were defined in the multi-level regression analyses: (i) student, (ii) class and (iii) school.

Mediation analyses were used to examine, at each of the three time points (8, 12 and 20 months), whether changes in water and diet drinks could explain the intervention effect on the consumption of sugar-sweetened beverages (outcome variable). A series of multi-level linear regression analyses were conducted ${ }^{(14)}$. The ANCOVA method was used to define changes between the baseline and post-intervention measurements as it corrects for the phenomenon of regression to the mean ${ }^{(15,16)}$.

First, the total effect of the DoiT intervention on the consumption of sugar-sweetened beverages was calculated (c-coefficient). In this regression model, the sugar-sweetened beverage outcome value post-intervention was adjusted for the baseline value. Second, the effect of the intervention on water and diet drinks (potential mediators) was calculated, adjusting for baseline values (a-coefficient). Third, the association between water and diet drinks and consumption of sugar-sweetened beverages, adjusting for baseline values for both the outcome and mediator variables, was calculated (b-coefficient). Analyses were conducted separately for each of the potential mediators.

The mediation effect (indirect effect) was estimated by calculating the product of the coefficients $(a \times b=a b)$ by multiplying the 'a-coefficient', representing the intervention effect on the mediators (i.e. consumption of water 
or diet drinks), with the 'b-coefficient', representing the relationship between the mediators (i.e. consumption of water or diet drinks) and sugar-sweetened beverage consumption. Standard errors were calculated and used to construct the $95 \% \mathrm{CI}$ using the Sobel test: $\mathrm{SE}=$ $\sqrt{ }\left(\mathrm{a}^{2} \times \mathrm{sE}_{\mathrm{b}}^{2}+\mathrm{b}^{2} \times \mathrm{sE}_{\mathrm{a}}^{2}\right)^{(14)}$. Finally, the proportion mediated ( $\%$ mediation), representing the amount of the intervention effect on changes in sugar-sweetened beverage consumption that could be explained by changes in water or diet drink consumption induced by the intervention, was calculated by dividing the indirect effect (ab) by the total effect $\left(a b+c^{\prime}\right)$, in which $c^{\prime}$ is the direct intervention effect on sugar-sweetened beverage consumption when controlled for the mediator. All analyses were adjusted for possible confounding by gender, age and ethnicity.

Variables that affect the hypothesised relationship among the variables (i.e. path a) are often known as moderators and are tested as interaction effects. In the present study, moderation analysis was performed to determine whether the effects differed for subgroups of participants regarding gender and ethnicity, by including an interaction term (e.g. intervention $\times$ gender) into the first and second regression analyses ${ }^{(17)}$ (Fig. 1).

\section{Results}

\section{Baseline characteristics}

Table 1 shows the characteristics of the sample at baseline for whom all data were available ( $n 747,50 \%$ boys). The mean age was 12.7 years and $88 \%$ were of Dutch or Western ethnicity.

Table 2 shows the baseline values for consumption of sugar-sweetened beverages (including fruit juice and soft drink consumption) and water and diet drinks for both the intervention and control groups. The majority of

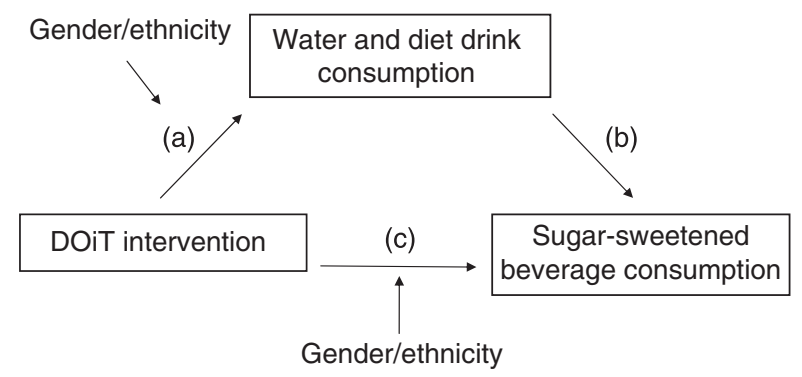

Fig. 1 Conceptual model: the Dutch Obesity Intervention in Teenagers (DOiT) intervention affects sugar-sweetened beverage consumption indirectly through water and diet drink consumption

Table 1 Characteristics at baseline for intervention and control groups

\begin{tabular}{|c|c|c|c|c|c|c|}
\hline \multirow[b]{2}{*}{ Characteristics } & \multicolumn{2}{|c|}{ Overall ( $n 747)$} & \multicolumn{2}{|c|}{ Intervention group ( $n$ 402) } & \multicolumn{2}{|c|}{ Control group (n 345) } \\
\hline & Mean & SD & Mean & SD & Mean & SD \\
\hline \multirow[t]{2}{*}{ Age (years) } & $12 \cdot 7$ & 0.5 & $12 \cdot 6$ & $0 \cdot 4$ & $12 \cdot 8$ & 0.5 \\
\hline & \multicolumn{2}{|c|}{$\%$} & \multicolumn{2}{|c|}{$\%$} & \multicolumn{2}{|c|}{$\%$} \\
\hline $\begin{array}{l}\text { Boys } \\
\text { Ethnicity }\end{array}$ & \multicolumn{2}{|c|}{$49 \cdot 5$} & \multicolumn{2}{|c|}{$47 \cdot 3$} & \multicolumn{2}{|c|}{$52 \cdot 2$} \\
\hline Dutch or Western ethnicity & \multicolumn{2}{|c|}{$87 \cdot 8$} & \multicolumn{2}{|c|}{$89 \cdot 3$} & \multicolumn{2}{|c|}{$86 \cdot 1$} \\
\hline Non-Western ethnicity (e.g. Turkish, Moroccan) & \multicolumn{2}{|c|}{$12 \cdot 2$} & \multicolumn{2}{|c|}{$10 \cdot 7$} & \multicolumn{2}{|c|}{$13 \cdot 9$} \\
\hline
\end{tabular}

Table 2 Consumption of sugar-sweetened beverages (fruit juice and soft drinks), water and diet drinks at baseline and percentage change from baseline at 8-, 12- and 20-month follow-ups

\begin{tabular}{|c|c|c|c|c|c|}
\hline & \multicolumn{2}{|c|}{ Baseline } & \multicolumn{3}{|c|}{ Percentage change from baseline } \\
\hline & Mean & SD & 8 months & 12 months & 20 months \\
\hline \multicolumn{6}{|c|}{ Sugar-sweetened beverages $(\mathrm{ml} / \mathrm{d})$} \\
\hline Intervention group & $1091 \cdot 6$ & $846 \cdot 4$ & $-29 \cdot 2$ & $-39 \cdot 3$ & $-40 \cdot 7$ \\
\hline Control group & $1154 \cdot 8$ & $874 \cdot 4$ & $-4 \cdot 7$ & $-17 \cdot 9$ & $-33 \cdot 8$ \\
\hline \multicolumn{6}{|l|}{ Fruit juice $(\mathrm{ml} / \mathrm{d})$} \\
\hline Intervention group & $327 \cdot 4$ & $365 \cdot 9$ & $-27 \cdot 5$ & $-35 \cdot 2$ & $-29 \cdot 6$ \\
\hline Control group & $375 \cdot 5$ & $408 \cdot 6$ & $-25 \cdot 5$ & $-24 \cdot 9$ & $-39 \cdot 3$ \\
\hline \multicolumn{6}{|l|}{ Soft drinks $(\mathrm{ml} / \mathrm{d})$} \\
\hline Intervention group & $829 \cdot 9$ & $657 \cdot 2$ & $-34 \cdot 7$ & $-44 \cdot 9$ & $-46 \cdot 6$ \\
\hline Control group & $881 \cdot 7$ & $723 \cdot 9$ & $-6 \cdot 9$ & $-23 \cdot 4$ & $-39 \cdot 2$ \\
\hline \multicolumn{6}{|l|}{ Water $(\mathrm{ml} / \mathrm{d})$} \\
\hline Intervention group & $440 \cdot 1$ & $474 \cdot 3$ & $16 \cdot 4$ & $0 \cdot 3$ & $3 \cdot 6$ \\
\hline Control group & $471 \cdot 3$ & $503 \cdot 7$ & $5 \cdot 8$ & $-2 \cdot 5$ & $-3 \cdot 8$ \\
\hline \multicolumn{6}{|l|}{ Diet drinks $(\mathrm{ml} / \mathrm{d})$} \\
\hline Intervention group & $164 \cdot 2^{*}$ & 273.9 & $-25 \cdot 5$ & $-35 \cdot 8$ & $-35 \cdot 1$ \\
\hline Control group & $221 \cdot 8$ & $316 \cdot 6$ & $-10 \cdot 3$ & $-36 \cdot 8$ & $-43 \cdot 8$ \\
\hline
\end{tabular}

*Mean value was significantly different from that of the control group $(P<0.01)$. 
sugar-sweetened beverage consumption was obtained from soft drinks. At baseline, the consumption of sugarsweetened beverages and water was comparable between the two groups. The intervention group consumed significantly fewer diet drinks $(164 \mathrm{ml} / \mathrm{d})$ than the control group $(222 \mathrm{ml} / \mathrm{d})$. The percentage change in the consumption of all beverages has been reported for all three time points.

Table 3 shows, at 8, 12 and 20 months, the intervention effect on sugar-sweetened beverages (c), the intervention effect on water and diet drinks (a), the association between water and diet drinks and sugar-sweetened beverages (b), the mediation effect (ab) and the proportion mediated.

\section{Intervention effects on sugar-sweetened beverages (c-coefficient)}

Immediately post intervention ( 8 months), the adolescents in the intervention group consumed significantly fewer sugar-sweetened beverages than adolescents in the control group $(-285 \mathrm{ml} / \mathrm{d} ; 95 \% \mathrm{CI}-421,-149)$. At the 12 -month follow-up, the difference in consumption was still significant, but decreased to $-260 \mathrm{ml} / \mathrm{d}(95 \% \mathrm{CI}-369,-160)$ and after 20 months, there was no significant difference in the consumption of sugar-sweetened beverages between groups. There was no interaction with gender or ethnicity at the 8-, 12- or 20-month follow-ups.

\section{Intervention effects on water and diet drinks (a-coefficient)}

At the 8-month follow-up, adolescents in the intervention group consumed significantly fewer diet drinks than the adolescents in the control group $(-52 \mathrm{ml} / \mathrm{d} ; 95 \% \mathrm{CI}-89$, -16). At this time point, the intervention also had a positive, but non-significant, effect on water consumption among the adolescents in the intervention group $(+47 \mathrm{ml} / \mathrm{d}$; $95 \% \mathrm{CI}$ $-27,121)$. There were no significant differences in water or diet drink consumption between adolescents in the intervention and control groups at the 12- or 20-month follow-ups. Gender and ethnicity were not effect modifiers.

\section{Association between water and diet drinks and sugar-sweetened beverages (b-coefficient)}

Water or diet drink consumption was not associated with the consumption of sugar-sweetened beverages at 8 months. There was a small but significant association between the consumption of diet drinks and sugar-sweetened beverages at the 12- and 20-month follow-ups.

\section{Mediation effect (ab-coefficient) and proportion mediated}

Neither water nor diet drinks appeared to mediate the intervention effect on sugar-sweetened beverage consumption at any time point.

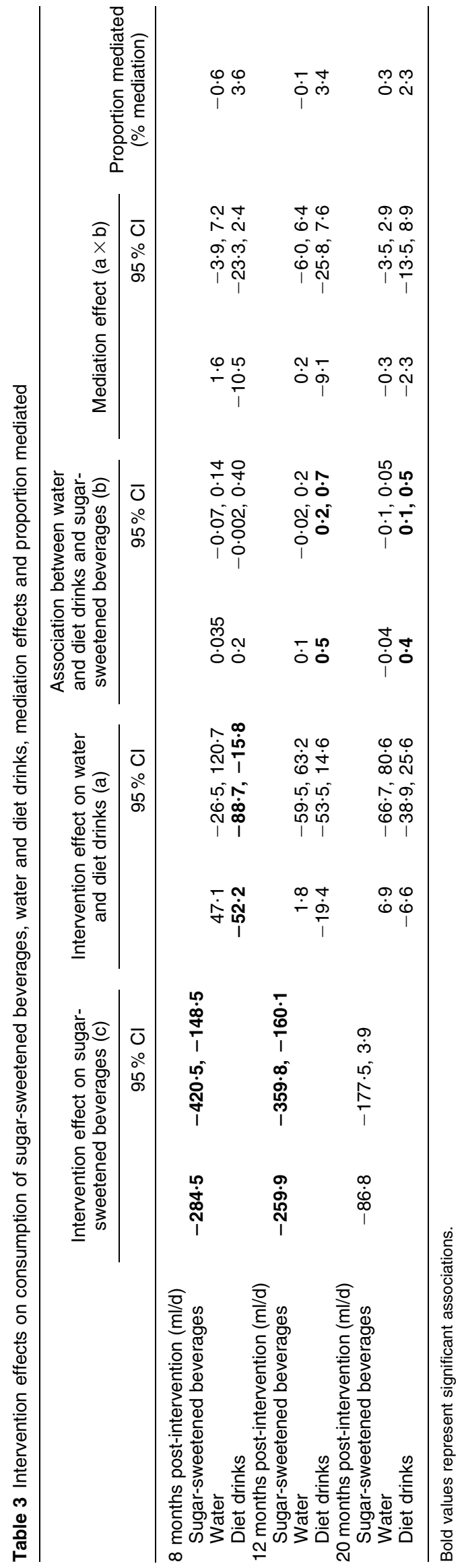




\section{Discussion}

The aim of the present study was to examine whether adolescents who decreased their consumption of sugarsweetened beverages, replaced their sugar-sweetened beverages with an increased consumption of water or diet drinks. The findings revealed that the DoiT intervention, which strongly promoted the reduction of sugar-sweetened beverages, resulted in a significant reduction in sugarsweetened beverages at the 8 -month $(-285 \mathrm{ml} / \mathrm{d})$ and 12-month $(-260 \mathrm{ml} / \mathrm{d})$ follow-ups; however, no significant differences were observed at 20 months (12 months postintervention). In the present study, sugar-sweetened beverages included soft drinks and fruit juice; however, the reduction was mainly due to a decrease in consumption of soft drinks. There was also a significant decrease in the consumption of diet drinks $(-52 \mathrm{ml} / \mathrm{d})$ at 8 months; however, this was not maintained at 12 or 20 months. No significant difference in water consumption between groups was observed at any time point.

We are aware of one other intervention study that reduced the consumption of total carbonated drinks (i.e. carbonated drinks with sugar, carbonated diet drinks and carbonated drinks with caffeine) among 7-11-year-old primary-school children by $150 \mathrm{ml}$ over $3 \mathrm{~d}^{(18)}$. In that study, the only follow-up measurement was immediately post-intervention, the magnitude of the effect was smaller, and in contrast to the current results, no significant reduction in carbonated drinks with sugar was observed. This intervention by James et $a l^{(18)}$ involved a younger age group, and focused only on the reduction of carbonated drinks, whereas the DOiT intervention also focused on other behaviours, such as snack consumption, sedentary behaviour and physical activity.

Although the DOiT intervention resulted in a decreased consumption of sugar-sweetened beverages, this could not be explained by the replacement or substitution with water and/or diet drinks. This is in contrast to what was hypothesised, as we expected that adolescents who decreased their consumption of sugar-sweetened beverages would replace this with water or diet drinks. The baseline consumption of sugar-sweetened beverages was, however, relatively high. It is therefore possible that there was substantial room for improvement without requiring replacement with other beverages, and that the overall fluid consumption was simply reduced.

These results indicate that targeting water and diet drinks may not be important or necessary in the interventions aimed at reducing the consumption of sugar-sweetened beverages among adolescents. It is also interesting to observe that the response to the intervention did not differ across gender and ethnicity and therefore it may not be necessary for future 'soft drink' interventions to be designed specifically for girls or boys or participants of different ethnicity. It is very likely that other mediators explained the intervention effect $^{(9)}$. Our findings also suggest that interventions aiming to reduce sugar-sweetened beverage consumption may be most effective by targeting soft drink rather than fruit juice intake. At present, there are no other studies to compare these results with as few mediation studies have been conducted for school-based nutrition intervention studies ${ }^{(19)}$, and to our knowledge, no sugarsweetened beverage intervention studies among adolescents have conducted mediation analysis.

The findings are limited by the fact that data collection relied exclusively on self-report. Another limitation may be that the measurement instruments were not sensitive enough to adequately measure changes in the target behaviours. It is also possible that the intervention may have assisted the participants in the intervention group to provide more socially desirable answers post-intervention. Further, although water intake was promoted throughout the DOiT intervention, unlike the promotion of reducing sugar-sweetened beverages, it was not one of the main messages. If the intervention included a concomitant promotion of water, a greater increase in water intake and replacement of sugar-sweetened beverages with water may have been observed. The strengths of the present study include the longitudinal randomized design, the presence of a control group and the presence of an intervention effect for the consumption of sugar-sweetened beverages.

\section{Conclusion}

Interventions aimed at reducing sugar-sweetened beverage consumption may be effective without also requiring change in the intake of other beverages. The present study provides useful findings that may help inform future interventions aimed at reducing intake of sugar-sweetened beverages among youth.

\section{Acknowledgements}

The present study is part of the Netherlands Health Foundation (NHF)-NRG, and was funded by the NHF (Grant no. 2000Z003), the Dutch Ministry of Health, Welfare and Sports and the Royal Association of Teachers and Physical Education. There are no conflicts of interest. J.V., A.S., M.M.v.S. and M.J.M.C. contributed to the study design, data analysis, interpretation of the results and writing of the manuscript. W.v.M. and J.B. contributed intellectual input to the overall study design and writing of the present paper. All authors read and approved the final manuscript. The authors thank all participants and schools involved in the study.

\section{References}

1. Singh AS, Mulder C, Twisk J et al. (2008) Tracking of childhood overweight into adulthood: a systematic review of the literature. Obes Rev 9, 474-488. 
2. Must A (2003) Does overweight in childhood have an impact on adult health? Nutr Rev 61, 139-142.

3. Rennie KL, Johnson L \& Jebb SA (2005) Behavioural determinants of obesity. Best Pract Res Clin Endocrinol Metab 19, 343-358.

4. Cavadini C, Siega-Riz AM \& Popkin B (2000) US adolescent food intake trends from 1965 to 1996. Arch Dis Child 83, $18-24$.

5. Vereecken C, Inchley J, Subramanian S et al. (2005) The relative influence of individual and contextual socio-economic status on consumption of fruit and soft-drinks among adolescents in Europe. Eur J Public Health 15, 224-232.

6. Vartanian L, Schwartz M \& Brownell K (2007) Effects of soft drink consumption on nutrition and health: a systematic review and meta-analysis. Am J Public Health 97, 667-675.

7. Nielsen J \& Popkin B (2004) Changes in beverage intake between 1977 and 2001. Am J Prev Med 27, 205-210.

8. Lien N, Lytle LA \& Klepp K (2001) Stability in consumption of fruit, vegetables and sugary food in a cohort from age 14 to age 21. Prev Med 33, 217-226.

9. ChinAPaw MJ, Singh AS, Brug J et al. (2008) Why did soft drink consumption decrease but screen time not? Mediating mechanisms in a school-based obesity prevention program. Int J Behav Nutr Phys Act 5, 41.

10. Singh AS, ChinAPaw MJ, Brug J et al. (2009) Dutch obesity intervention in teenagers: effectiveness of a school-based program on body composition and behavior. Arch Pediatr Adoles Med 163, 307-317.

11. Singh AS, ChinAPaw MJ, Kremers SP et al. (2006) Design of the Dutch Obesity Intervention in Teenagers (NRG-DOiT): systematic development, implementation and evaluation of a school-based intervention aimed at the prevention of excessive weight gain in adolescents. BMC Public Health 6, 304 .

12. van Assema P, Brug J, Ronda G et al. (2001) The relative validity of a short Dutch questionnaire as a means to categorize adults and adolescents to total and saturated fat intake. J Hum Nutr Diet 14, 377-390.

13. van Assema P, Brug J, Ronda G et al. (2002) A short Dutch questionnaire to measure fruit and vegetable intake: relative validity among adults and adolescents. Nutr Health Forum 16, 85-106.

14. MacKinnon DP (editor) (2008) Introduction to Statistical Mediation Analyses. New York: Taylor \& Francis Group.

15. Twisk J \& Proper K (2004) Evaluation of the results of a randomized controlled trial: how to define changes between baseline and follow-up. J Clin Epidemiol 57, 223-228.

16. Vickers AJ \& Altman DG (2001) Analysing controlled trials with baseline and follow up measurements. BMJ 323, 1123-1124.

17. MacKinnon DP, Fairchild AJ \& Fritz MS (2007) Mediation analysis. Annu Rev Psychol 58, 593-614.

18. James J, Thomas P, Cavan D et al. (2004) Preventing childhood obesity by reducing consumption of carbonated drinks: cluster randomised controlled trial. BMJ 328, 1237.

19. Cerin E \& MacKinnon D (2009) A commentary on current practice in mediating variable analyses in behavioural nutrition and physical activity. Public Health Nutr 12, 1182-1188. 